\title{
Noninvasive assessment of isocitrate dehydrogenase mutation status in cerebral gliomas by magnetic resonance spectroscopy in a clinical setting
}

\author{
Anna Tietze, MD, PhD,, 2 Changho Choi, PhD, ${ }^{3}$ Bruce Mickey, MD, ${ }^{4}$ Elizabeth A. Maher, MD, PhD, ${ }^{5}$ \\ Benedicte Parm Ulhøi, MD, ${ }^{6}$ Ryan Sangill, MSc, PhD, ${ }^{1,2}$ Yasmin Lassen-Ramshad, MD, PhD, ${ }^{7}$ \\ Slavka Lukacova, MD, PhD, ${ }^{7}$ Leif Østergaard, MD, PhD, DSc, ${ }^{1,2}$ and Gorm von Oettingen, MD, PhD ${ }^{8}$ \\ Departments of ${ }^{1}$ Neuroradiology, ${ }^{6}$ Pathology, ${ }^{7}$ Oncology, and ${ }^{8}$ Neurosurgery, Aarhus University Hospital; ${ }^{2}$ Center of Functionally \\ Integrative Neuroscience, Aarhus University, Aarhus, Denmark; and ${ }^{3}$ Advanced Imaging Research Center-Radiology, \\ ${ }^{4}$ Neurological Surgery Clinic, and ${ }^{5}$ Departments of Internal Medicine and Neurology \& Neurotherapeutics, University of Texas \\ Southwestern Medical Center, Dallas, Texas
}

OBJECTIVE Mutations in the isocitrate dehydrogenase (IDH) genes are of proven diagnostic and prognostic significance for cerebral gliomas. The objective of this study was to evaluate the clinical feasibility of using a recently described method for determining IDH mutation status by using magnetic resonance spectroscopy (MRS) to detect the presence of 2-hydroxyglutarate $(2 \mathrm{HG})$, the metabolic product of the mutant IDH enzyme.

METHODS By extending imaging time by 6 minutes, the authors were able to include a point-resolved spectroscopy (PRESS) MRS sequence in their routine glioma imaging protocol. In 30 of 35 patients for whom this revised protocol was used the lesions were subsequently diagnosed histologically as gliomas. Of the remaining 5 patients, 1 had a gangliocytoma, 1 had a primary CNS lymphoma, and 3 had nonneoplastic lesions. Immunohistochemistry and/or polymerase chain reaction were used to detect the presence of IDH mutations in the glioma tissue resected.

RESULTS In vivo MRS for $2 \mathrm{HG}$ correctly identified the IDH mutational status in $88.6 \%$ of patients. The sensitivity and specificity was $89.5 \%$ and $81.3 \%$, respectively, when using $2 \mathrm{mM} 2 \mathrm{HG}$ as threshold to discriminate IDH-mutated from wildtype tumors. Two glioblastomas that had elevated $2 \mathrm{HG}$ levels did not have detectable IDH mutations, and in 2 IDHmutated gliomas $2 \mathrm{HG}$ was not reliably detectable.

CONCLUSIONS The noninvasive determination of the IDH mutation status of a presumed glioma by means of MRS may be incorporated into a routine diagnostic imaging protocol and can be used to obtain additional information for patient care.

https://thejns.org/doi/abs/10.3171/2016.10.JNS161793

KEY WORDS glioma; MRI; isocitrate dehydrogenase; IDH mutations; MR spectroscopy; oncology

$\mathrm{M}$ UTATIONS in the isocitrate dehydrogenase (IDH) genes $I D H I$ and $I D H 2$ in cerebral gliomas have drawn great interest in recent years, as they are an important diagnostic and prognostic biomarker. ${ }^{11}$ The Cancer Genome Atlas Network (http://cancergenome. nih.gov/) showed already in 2010 that patients with IDH-mutated glioblastoma (GBM) have a more favorable outcome than those with wildtype tumors, ${ }^{22}$ and the recently published update of the WHO brain tumor classification defines IDH mutation status as a key ge- netic feature in the current glioma classification. ${ }^{16}$ The prognostic value of IDH mutations for low-grade gliomas is currently less clear, ${ }^{1}$ but it has been established that wildtype tumors show a clinical behavior that resembles that of high-grade rather than low-grade gliomas., ${ }^{5,16}$ The underlying epigenetic and direct metabolic consequences of these mutations remain to be elucidated. ${ }^{14,24}$ IDH mutations and downstream metabolites are, however, already therapeutically targeted, and clinical trials are currently ongoing. ${ }^{18,19}$

ABBREVIATIONS AUC = area under the curve; CRLB = Cramér-Rao lower bound; FWHM = full width at half maximum; GBM = glioblastoma; IDH = isocitrate dehydrogenase; $M R S=$ MR spectroscopy; MS = multiple sclerosis; PCNSL = primary CNS lymphoma; ppm = parts per million; $P C R=$ polymerase chain reaction; PRESS = pointresolved spectroscopy; ROC = receiver operating characteristic; STEAM = stimulated echo acquisition mode; TE = echo time; $2 \mathrm{HG}=2$-hydroxyglutarate.

SUBMITTED July 10, 2016. ACCEPTED October 14, 2016.

INCLUDE WHEN CITING Published online March 3, 2017; DOI: 10.3171/2016.10.JNS161793. 
The usual diagnostic workup for determination of the presence of IDH mutations is tissue analysis by immunohistochemistry with antibodies against the most common mutant protein, $\mathrm{R} 132 \mathrm{H}$, or polymerase chain reaction (PCR)-based methods that allow the detection of mutations in the $I D H 1$ and $I D H 2$ genes (R172K). These approaches obviously require sampling of glioma tissue, which is accomplished by surgical biopsy or tumor resection.

Magnetic resonance spectroscopy (MRS) may provide a noninvasive determination of the IDH-mutation status. ${ }^{2,3,6,8,10,17}$ The mutant IDH enzyme is metabolically active and produces 2-hydroxyglutarate (2HG), which is thought to be a potent oncometabolite. As a result, 2HG, which is present in very low quantities (micromolar levels) in normal cells or IDH wildtype cells, is increased by orders of magnitude in IDH-mutated cells. Previous studies have demonstrated that increased 2HG levels in IDH-mutated glioma tissue are detectable by means of MRS, and the presence of $2 \mathrm{HG}$ on MRS can serve as a biomarker for the IDH-mutation status of a glioma. Several MRS techniques have been described, including standard short echo time (TE) point-resolved spectroscopy (PRESS), ${ }^{17} 2 \mathrm{HG}-$ tailored long TE PRESS, ${ }^{6,8,10} \mathrm{~J}$-difference editing MRS, ${ }^{2,3,8}$ and 2D-correlation spectroscopy (COSY). ${ }^{2}$ The $2 \mathrm{HG}$ protons resonate at different locations of the spectrum, with the largest peak at approximately $2.25 \mathrm{ppm}$ (parts per million) at $3 \mathrm{~T} .{ }^{8}$ Initial results are encouraging, showing a high correlation between the presence of $2 \mathrm{HG}$ on MRS and the IDH mutation status from tissue analysis. ${ }^{8,10}$

The noninvasive determination of IDH-mutation status may be clinically useful in many regards. First, it allows the differentiation of gliomas from solitary metastases, primary cerebral lymphoma, or nonneoplastic lesions and may be beneficial in tumor locations associated with highrisk surgery. Second, it may influence surgical decisionmaking regarding the extent of resection, as aggressive resection of both enhancing and nonenhancing disease might improve survival in IDH-mutated, but not in wildtype, tumors. ${ }^{4}$ Third, 2 HG MRS has shown promise for monitoring treatment response. Successful cytoreductive therapy leads to a decrease of $2 \mathrm{HG}$-producing tumor cells, whereas tumor progression causes $2 \mathrm{HG}$ increases., ${ }^{7,9,10}$ Lastly, when IDH mutations or downstream metabolites are going to be used as treatment targets, 2HG MRS will be an important tool to demonstrate therapy response or failure.

The clinical implementation of the long TE PRESS technique at $3 \mathrm{~T}^{8}$ is therefore highly relevant. New imaging methods, particularly if applied in a multicenter setting, require fast, reliable, and reproducible approaches, and we therefore tested this previously reported PRESS sequence to determine the presurgical IDH status in suspected cerebral gliomas and compared our results to the gold standard of immunohistochemistry and PCR. We performed single-voxel MRS as part of our routine imaging protocol. The primary focus of our study was to evaluate this technique in a busy clinical department, where time constraints are a common issue. Therefore a single MRS measurement was performed without resampling in different tumor regions.

\section{Methods Patients}

A total of 35 patients (14 female, 21 male) were included in this study, which was approved by the National Committee on Health Research Ethics and Data Protection Agency. Inclusion criteria were the suggestion of a cerebral glioma on primary imaging by CT or MRI, age between 18 and 65 years, and the ability to give informed consent.

\section{MRI Acquisition}

Imaging was performed on a 3-T Philips Achieva system with a standard 8-channel reception head coil. The MRI protocol consisted of 3D T1-weighted images acquired before and after intravenous administration of a contrast agent (TR $8.4 \mathrm{msec}$, TE $4 \mathrm{msec}$, voxel size 0.94 $\times 0.94 \times 1 \mathrm{~mm}^{3}$, FOV $240 \times 240 \mathrm{~mm}^{2}$, scan duration 5 minutes 5 seconds); sagittal T2 TSE (TR $3000 \mathrm{msec}$, TE $80 \mathrm{msec}$, voxel size $0.43 \times 0.43 \times 4 \mathrm{~mm}^{3}$, FOV 240 $\times 231 \mathrm{~mm}^{2}$, scan duration 1 minute 39 seconds); axial T2 FLAIR (TR 11,000 msec, TE $125 \mathrm{msec}$, TI $2800 \mathrm{msec}$, voxel size $0.45 \times 0.45 \times 4 \mathrm{~mm}^{3}$, FOV $230 \times 183 \mathrm{~mm}^{2}$, scan duration 5 minutes 8 seconds); dynamic susceptibility contrast perfusion-weighted MRI (2D gradient-echo, TR $1200 \mathrm{msec}$, TE $30 \mathrm{msec}$, voxel size $1.6 \times 1.6 \times 4 \mathrm{~mm}^{3}, 100$ dynamic images, scan duration 2 minutes 4 seconds); diffusion-weighted imaging (echo planar imaging, TR 4024 msec, TE $99 \mathrm{msec}$, voxel size $1.2 \times 1.2 \times 4 \mathrm{~mm}^{3}$, FOV 230 $\times 230 \mathrm{~mm}^{2}$, scan duration 1 minute 24 seconds). For the perfusion-weighted MRI, a standard intravenous dose of $0.1 \mathrm{mmol} / \mathrm{kg}$ gadobutrol was administered, which subsequently was used for postcontrast T1-weighted series.

For MRS, an optimized PRESS sequence was used as described in prior studies: $6,7 \mathrm{TR} 2000 \mathrm{msec}, \mathrm{TE}_{1} 32 \mathrm{msec}$, $\mathrm{TE}_{2} 65 \mathrm{msec}$, voxel size $2 \times 2 \times 2 \mathrm{~cm}^{3}, 128$ repetitions, scan duration 4 minutes 22 seconds. A neuroradiologist (with 8 years of experience) placed the voxel prior to contrast administration, avoiding necrotic, hemorrhagic, or calcified tumor regions and using axial T2 FLAIR, sagittal T2-weighted, and 3D T1-weighted images for guidance (Fig. 1). In addition, an unsuppressed water signal was acquired from the PRESS MRS voxel using stimulated echo acquisition mode (STEAM): TR 20,000 msec, TE $9.1 \mathrm{msec}$, TM $16 \mathrm{msec}$, voxel size $2 \times 2 \times 2 \mathrm{~cm}^{3}, 1$ repetition, scan duration 40 seconds. Corrections for eddy current effects and frequency drifts were conducted using vendor-supplied functions in the scanner. The overall imaging time was approximately 30 minutes.

\section{MRS Postprocessing for 2HG Quantification}

A neuroradiologist performed the postacquisition MRS data processing before surgery. The analysis was conducted offline using in-house MATLAB scripts on a local PC (Mathworks). MRS spectra were apodized with a 0.5$\mathrm{Hz}$ exponential function prior to Fourier transformation. Spectral fitting was conducted between 0.5 and $4.1 \mathrm{ppm}$ using LCModel (http://lcmodel.ca) (for details see Choi et al. $\left.{ }^{6}\right)$. The $2 \mathrm{HG}$ concentration was obtained with reference to unsuppressed STEAM water signal, similarly as in a prior study. ${ }^{6}$ The overall postprocessing time per patient 

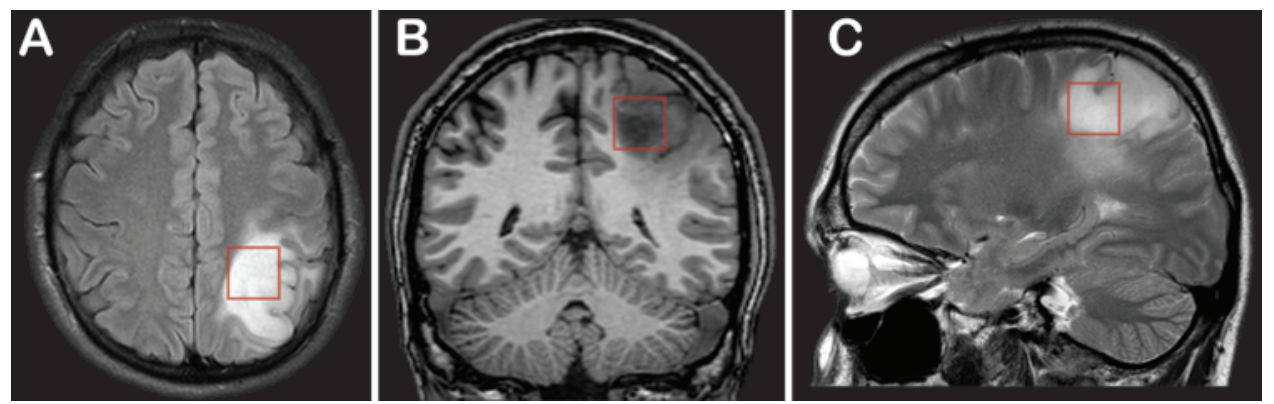

FIG. 1. Axial T2 FLAIR (A), coronal T1-weighted (B), and sagittal T2-weighted (C) images suggesting a glioma in the left parietal lobe. The red boxes indicate the location of the MRS voxel. The $2 \mathrm{HG}$ concentration, measured by MRS, was $3.56 \mathrm{mM}$. Histological examination showed a Grade II astrocytoma. PCR detected a mutation in the IDH1 gene (R132H). Figure is available in color online only.

was approximately 2 minutes. A $2 \mathrm{HG}$ concentration of 2 $\mathrm{mM}$ was defined as a threshold for IDH mutation..$^{8,9}$

The quality of the fitting procedure was assessed by calculating the Cramér-Rao lower bound (CRLB). Values with a CRLB $<20 \%$ were viewed as being reliable. The quality of the PRESS data were evaluated using the full width at half maximum (FWHM) of the choline peak returned by LCModel, and spectra with FWHM $<10 \mathrm{~Hz}$ were rated as being diagnostic.

\section{Image-Guided Stereotactic Surgical Biopsies}

To correlate imaging data and results from immunohistochemistry and/or PCR directly, biopsies were obtained from the tumor regions sampled by MRS in all patients who underwent surgery. The MRS voxel and the postcontrast T1-weighted image data were co-registered offline and transferred to the image-guided neurosurgery system (StealthStation S7, Medtronic). The contrast of the MRS voxel was adjusted, such that essential details of the structural image remained clearly visible. A craniotomy was performed, and tumor tissue was obtained prior to tumor resection. We used a standard needle (outer diameter of $2.2 \mathrm{~mm}$ ) to approach the MRS voxel by applying the optical image-guided Vertek Medtronic biopsy solution.

\section{Immunohistochemistry and PCR Analysis of Glioma Tissue}

Immunohistochemical analysis was performed for mutated IDHI (R132H) protein expression in all glioma cases, using standard techniques with the Benchmark XT automatic staining platform (Ventana Medical Systems). Briefly, 4- $\mu \mathrm{m}$ sections of formalin-fixed, paraffin-embedded tissue were cut on coated glass slides, deparaffinized, and rehydrated. Heat-induced epitope retrieval was performed with Ventana $\mathrm{CCl}$ at $\mathrm{pH}$ 9. Slides were incubated for 32 minutes with anti-IDH1 (R132H) mouse monoclonal antibody (clone H09, Dianova), diluted at 1:20. Signals were detected using the ultraView Universal DAB Detection Kit (Ventana Medical Systems), and slides counterstained with hematoxylin. Sections from an IDHI (R132H)-mutated glioma were used as a positive control, whereas negative control was performed by omitting the primary antibody. Additionally, endothelial cells were used as an internal negative control.
If immunohistochemistry results were equivocal or suggested wildtype IDH mutational status, this was either confirmed or refuted by PCR. ${ }^{16}$ DNA was extracted from formalin-fixed, paraffin-embedded, and deparaffinized tissue using the QIAsymphony DSP DNA Mini Kit (Qiagen). Mutations in $I D H I$ or $I D H 2$ genes were detected with real-time PCR, using the therascreen IDHI/2 RGQ PCR Kit (Qiagen) according to the manufacturer's instructions. This assay detects 12 common IDHI/2 mutations (6 within codon 132 of the IDHI gene, 5 within codon 172 of the $I D H 2$ gene, 1 within codon 100 of the $I D H 1$ gene). PCR was performed with $25 \mathrm{ng}$ of sample or control DNA.

\section{Statistical Analysis}

Statistical analysis was performed using RStudio. A receiver operating characteristic (ROC) curve was constructed to determine the diagnostic performance of MRS, measured by the area under the curve (AUC). The sensitivity, specificity, positive predictive value, and negative predictive value for $2 \mathrm{HG}$ concentrations discriminating IDH-mutated from wildtype tumors were determined.

\section{Results \\ Patients}

Patient characteristics are reported in Table 1. The T2 FLAIR hyperintensity lesion volume was between 0.52 and $140.83 \mathrm{~cm}^{3}$ (mean $\left.77.88 \pm 39.99 \mathrm{~cm}^{3}\right)$. Histopathological diagnosis was obtained in 32 of 35 cases: 8 cases of Grade II astrocytoma, 5 of Grade III astrocytoma, 11 of glioblastoma (GBM), 6 of Grade III oligodendroglioma, 1 of gangliocytoma, and 1 of primary CNS lymphoma (PCNSL). In 2 of the remaining cases, the obtained tissue contained nonspecific inflammatory cells and normal neurons. No definite diagnosis could be found in one of these patients (a 19-year-old man), whereas the diagnosis of tumefactive multiple sclerosis (MS) was made on the basis of serial MRI scans in the other patient (a 22-yearold woman). In the last case, biopsy was deferred, and an autoimmune encephalitis was diagnosed based on the clinical course and a typical cerebrospinal fluid profile.

\section{IDH Mutational Status Determined by Immunohistochemistry and/or PCR}

In the 34 biopsy cases, immunohistochemistry was 
TABLE 1. Diagnosis, T2 FLAIR tumor volume, patient age, and immunohistochemistry and PCR results in all 35 cases

\begin{tabular}{|c|c|c|c|c|c|}
\hline Diagnosis & $\mathrm{IHC}$ & PCR & 2HG Concentration ( $\mathrm{mM} \pm \mathrm{SD}$ ) & Mean T2 FLAIR Hyperintensity Vol (ml) & Age \\
\hline A2 & Equivocal & $\mathrm{R} 132 \mathrm{C}$ & $3.66 \pm 0.40$ & 79.77 & $43 \mathrm{yrs}, 10 \mathrm{mos}$ \\
\hline A2 & Equivocal & $\mathrm{R} 132 \mathrm{H}$ & $4.77 \pm 0.29$ & 36.75 & $19 \mathrm{yrs}, 1 \mathrm{mo}$ \\
\hline A2 & Positive & NA & $0.45 \pm 0.18$ & 90.79 & $47 \mathrm{yrs}, 4 \mathrm{mos}$ \\
\hline A2 & Positive & NA & $0.35 \pm 0.28$ & 122.73 & 31 yrs, 0 mos \\
\hline A2 & Positive & NA & $2.98 \pm 0.21$ & 9.94 & $50 \mathrm{yrs}, 11 \mathrm{mos}$ \\
\hline A2 & Negative & $\mathrm{R} 132 \mathrm{C}$ & $5.69 \pm 0.23$ & 77.86 & $26 \mathrm{yrs}, 5 \mathrm{mos}$ \\
\hline A2 & Positive & $\mathrm{R} 132 \mathrm{H}$ & $3.45 \pm 0.24$ & 46.16 & $29 \mathrm{yrs}, 7 \mathrm{mos}$ \\
\hline A2 & Positive & NA & $8.03 \pm 0.32$ & 60.31 & $48 \mathrm{yrs}, 6 \mathrm{mos}$ \\
\hline A3 & Positive & NA & $1.17 \pm 0.31$ & 63.05 & 39 yrs, 1 mo \\
\hline A3 & Positive & $\mathrm{R} 132 \mathrm{H}$ & $2.69 \pm 0.32$ & 41.77 & $37 \mathrm{yrs}, 5 \mathrm{mos}$ \\
\hline A3 & Equivocal & $\mathrm{R} 132 \mathrm{C}$ & $8.71 \pm 0.44$ & 69.44 & $40 \mathrm{yrs}, 11 \mathrm{mos}$ \\
\hline A3 & Equivocal & $\mathrm{R} 132 \mathrm{H}$ & $2.93 \pm 0.23$ & 125.97 & $28 \mathrm{yrs}, 6 \mathrm{mos}$ \\
\hline A3 & Positive & NA & $3.01 \pm 0.24$ & 65.88 & $18 \mathrm{yrs}, 5 \mathrm{mos}$ \\
\hline GBM & Positive & NA & $13.93 \pm 0.28$ & 102.71 & $42 \mathrm{yrs}, 7 \mathrm{mos}$ \\
\hline GBM & Negative & Negative & $1.55 \pm 0.26$ & 70.62 & 39 yrs, 5 mos \\
\hline GBM & Negative & Negative & $2.0 \pm 0.46$ & 125.96 & $47 \mathrm{yrs}, 7 \mathrm{mos}$ \\
\hline GBM & Negative & Negative & $5.11 \pm 0.31$ & 111.52 & $62 \mathrm{yrs}, 11 \mathrm{mos}$ \\
\hline GBM & Negative & Negative & $1.7 \pm 0.24$ & 107.33 & $58 \mathrm{yrs}, 6 \mathrm{mos}$ \\
\hline GBM & Negative & Negative & $0.6 \pm 0.23$ & 130.25 & 59 yrs, 7 mos \\
\hline GBM & Negative & Negative & $0.33 \pm 0.39$ & 60.41 & $64 \mathrm{yrs}, 6 \mathrm{mos}$ \\
\hline GBM & Negative & Negative & $1.76 \pm 0.28$ & 62.41 & $69 \mathrm{yrs}, 1 \mathrm{mo}$ \\
\hline GBM & Negative & Negative & ND & 80.63 & 59 yrs, 4 mos \\
\hline GBM & Negative & Negative & $2.39 \pm 0.34$ & 127.33 & 44 yrs, 2 mos \\
\hline GBM & Positive & NA & $5.59 \pm 028$ & 89.87 & $25 \mathrm{yrs}, 10 \mathrm{mos}$ \\
\hline $\mathrm{O} 3$ & Negative & $\mathrm{R} 132 \mathrm{C}$ & $4.06 \pm 0.28$ & 128.63 & $42 \mathrm{yrs}, 10 \mathrm{mos}$ \\
\hline 03 & Negative & Negative & $0.88 \pm 0.48$ & 112.44 & $50 \mathrm{yrs}, 5 \mathrm{mos}$ \\
\hline 03 & Negative & Negative & $0.41 \pm 0.22$ & 42.58 & $47 \mathrm{yrs}, 7 \mathrm{mos}$ \\
\hline O3 & Positive & R312C & $4.12 \pm 0.25$ & 140.83 & $66 \mathrm{yrs}, 0 \mathrm{mos}$ \\
\hline 03 & Positive & NA & $3.33 \pm 0.23$ & 54.45 & 46 yrs, 8 mos \\
\hline 03 & Negative & R172K & $4.80 \pm 0.34$ & 128.79 & $36 \mathrm{yrs}, 7 \mathrm{mos}$ \\
\hline Gangliocytoma & NA & NA & $1.32 \pm 0.21$ & 3.50 & 19 yrs, 9 mos \\
\hline Tumefactive MS & NA & NA & $1.67 \pm 0.20$ & 81.84 & 22 yrs, 3 mos \\
\hline Nonspecific inflammation & NA & NA & $1.90 \pm 0.49$ & 0.52 & $19 \mathrm{yrs}, 1 \mathrm{mo}$ \\
\hline PCNSL & NA & NA & $0.49 \pm 0.21$ & 28.08 & $35 \mathrm{yrs}, 1 \mathrm{mo}$ \\
\hline Autoimmune encephalitis & NA & NA & $0.63 \pm 0.41$ & 32.50 & $54 \mathrm{yrs}, 11 \mathrm{mos}$ \\
\hline
\end{tabular}

A2 = astrocytoma, Grade II; A3 = astrocytoma, Grade III; IHC = immunohistochemistry; NA = not available; ND = not detectable; O3 = oligodendroglioma, Grade III. False-negative and false-positive cases are in boldface type.

performed in 30 specimens and PCR in 21. IDH status was not analyzed in the patients diagnosed with gangliocytoma, PCNSL, or tumefactive MS or in the patient with nonspecific inflammation. Mutations in the IDHI gene were found in 18 of 34 patients, whereas we had only 1 case with an IDH2 mutation. IDH/R132H immunostaining was positive in 12 tumors, equivocal in 4 tumors, and negative in 14 tumors. Of the 18 tumors with equivocal or negative results, further examination with PCR demonstrated R132C mutations in 5, R132H in 4, and R172K in 1 tumor. The remaining 11 tumors were negative for IDH mutations.

\section{HG MRS and Sensitivity and Specificity for IDH Mutation Status}

The average quality of the PRESS data were good in the 35 patients (FWHM $[ \pm \%$ SD]: $6.32 \mathrm{~Hz}[ \pm 3.63 \mathrm{~Hz}])$.
The FWHM was large in 4 cases $(13.82,12.14,10.35$, and $10.25 \mathrm{~Hz}$ ). In 19 of 35 cases the lesions were diagnosed as IDH-mutated tumors based on MRS, with an average 2HG concentration of $4.82 \mathrm{mM}$. One case showed a borderline $2 \mathrm{HG}$ concentration of $2 \mathrm{mM}$ (CRLB 23\%) and was counted as wildtype.

Although not definitely proven, wildtype IDH was assumed for the gangliocytoma, PCNSL, tumefactive MS, and autoimmune encephalitis as well as for the case with no definite diagnosis (as it showed normal neurons and inflammation), since no mutations in these diseases have been identified to date. ${ }^{12}$ The IDH mutation status was therefore either known from tissue samples or was concluded from the final diagnosis in all cases.

MRS diagnosed the IDH mutation status correctly in 31 of 35 patients. Two cases were false negative (both astrocytoma, one Grade II and one Grade III) and 2 cases 

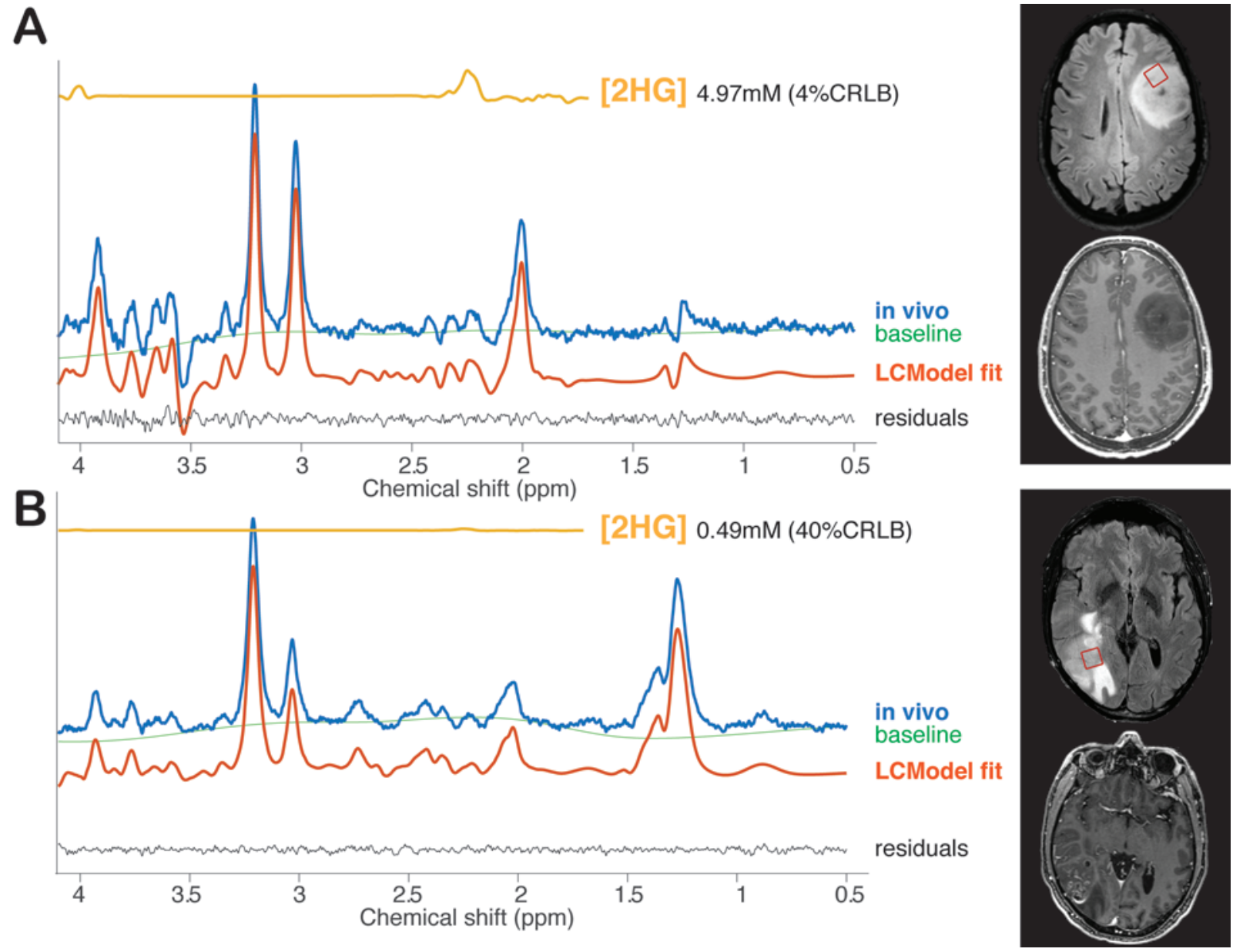

FIG. 2. A: MRS results and axial T2 FLAIR (upper) and postcontrast T1-weighted (lower) MR images obtained in a 27-year-old man with a Grade II astrocytoma. MRS shows a peak at $2.25 \mathrm{ppm}$, where $2 \mathrm{HG}$ protons are expected to resonate. The $2 \mathrm{HG}$ concentration is estimated to be $4.97 \mathrm{mM}( \pm 4 \% \mathrm{CRLB})$. PCR verified a mutation in the IDH1 gene (R132C). B: MRS results and axial T2 FLAIR (upper) and postcontrast T1-weighted (lower) MR images obtained a 59-year-old man with a wildtype GBM, shown for comparison. A subthreshold $2 \mathrm{HG}$ concentration of $0.49 \mathrm{mM}( \pm 40 \% \mathrm{CRLB})$ is in agreement with a nonmutated glioma (proven by immunohistochemistry and PCR). The red boxes indicate the location of the MRS voxels.

were false positive (both GBM), when compared with IDH mutation status diagnosed by immunohistochemistry or PCR. FWHM was $<10 \mathrm{~Hz}$ in all of these cases.

Representative MRS examples obtained in a patient with an IDH-mutated grade and a patient with a GBM are shown in Fig. 2A and B. The IDH mutational status was confirmed by PCR in both cases. A peak at $2.25 \mathrm{ppm}$, assigned to $2 \mathrm{HG}$, is clearly discernible in Fig. 2A, whereas a subthreshold $2 \mathrm{HG}$ concentration was detected in the wildtype GBM (Fig. 2B).

The $2 \mathrm{HG}$ concentrations are demonstrated as bar plots in Fig. 3. Figure 4 shows the ROC curve for detecting IDH mutation status by means of MRS. The AUC was 0.88 . The sensitivity, specificity, positive predictive, and negative predictive values for a cutoff value of $2-\mathrm{mM}$ 2HG were $89.5 \%, 81.3 \%, 89.5 \%$, and $87.5 \%$, respectively. Postcontrast T1-weighted images and the corresponding MRS voxels of the false-positive cases are displayed in Fig. 5A and B. False-negative cases are shown in Fig. 5C and $\mathrm{D}$.

\section{Discussion}

We have investigated the clinical feasibility and reliability of 2HG MRS for the detection of IDH mutations in glioma tissue. Previous studies have primarily been conducted in a research setting, ,,6,8,9 whereas the aim of our study was to assess the reliability of this technique on a standard 3-T MR system as part of a routine tumor imaging protocol. As shortage of time is a common issue in busy clinical departments, we only acquired 1 single-voxel MRS per patient. We found agreement between $2 \mathrm{HG}$ MRS and IDH mutation status, assessed by immunohistochemistry and/or PCR, in about $88 \%$ of our cases, which is encouraging, considering that the additional imaging and postprocessing time was only about 8 minutes per patient. However, our results did not reach the same sensitivity as reported in the 2 previous pilot studies. ${ }^{2,8}$ The reliability of $2 \mathrm{HG}$ MRS can be improved by increasing the number of measurements per voxel (in our case 128 repetitions), by resampling in different tumor regions, and by decreas- 


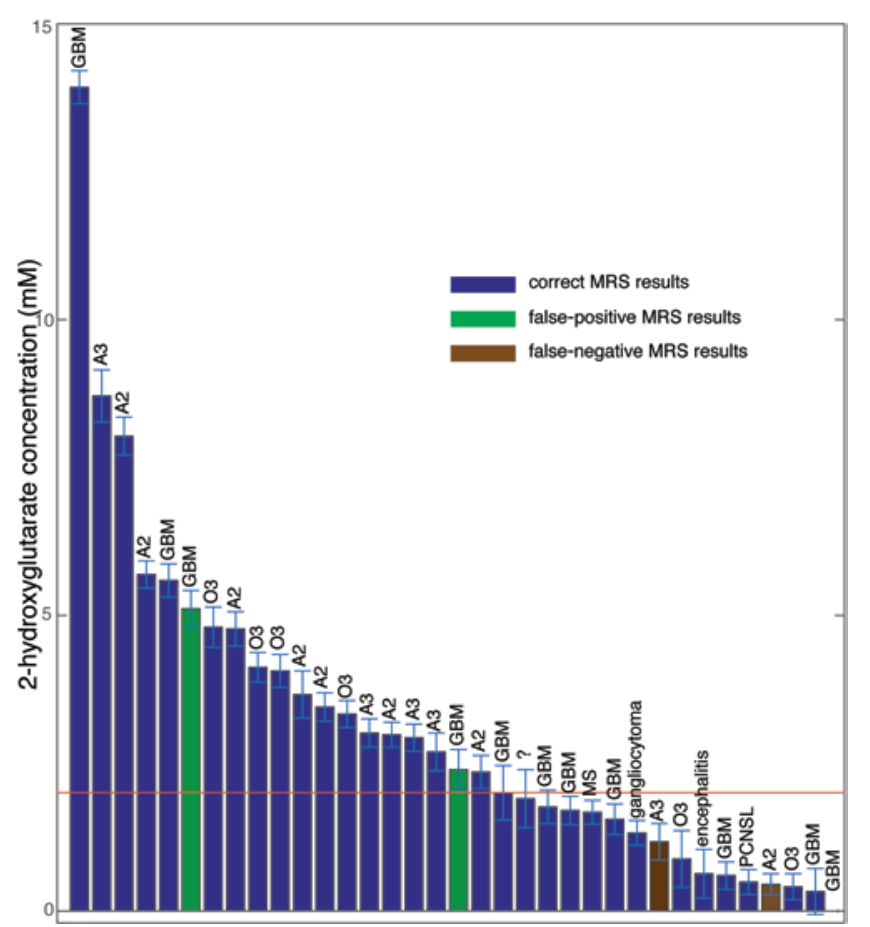

FIG. 3. The height of the bars represents the estimated $2 \mathrm{HG}$ concentration in $\mathrm{mM}$ (SD indicated by light blue error bars). The red line indicates the threshold of $2 \mathrm{mM}$ defining tumor tissue as being either IDH mutated $(>2 \mathrm{mM})$ or as being wildtype $(<2 \mathrm{mM})$. Correct MRS results are shown as blue bars, false positive as green bars, and false negative as brown bars. $\mathrm{A} 2$ = astrocytoma, Grade II; A3 = astrocytoma, Grade III; GBM = glioblastoma; $\mathrm{MS}$ = tumefactive multiple sclerosis; $\mathrm{O3}=$ oligodendroglioma, Grade III; PCNSL = primary CNS lymphoma.

ing the voxel size to avoid nontumor tissue, all of which contribute to an extended imaging time. In our approach, we had to limit scan duration maximally, which is likely to have influenced our results. The sensitivity for detecting IDH mutations by MRS in our study is higher than that reported by de la Fuente al., ${ }^{10}$ who recently tested $2 \mathrm{HG}$ MRS as part of a clinical tumor protocol and found correlation between MRS and IDH mutation in $48 \%$ of their cases. They underscore the importance of voxel size versus tumor volume, as they found that $2 \mathrm{HG}-\mathrm{MRS}$-positivity was rare in small tumors with a maximum dimension $<2 \mathrm{~cm}$. In these cases, the MRS voxel might be contaminated by normal brain tissue, which reduces the intravoxel $2 \mathrm{HG}$ concentration. Reducing the voxel size and increasing the number of signal averages can compensate for this, but it limits the applicability of the technique in clinics considerably due to time constraints and the risk of motion artifacts. The higher sensitivity in our study can be explained by an overall voxel size of $8 \mathrm{~cm}^{3}$ versus a volume of $\leq 8$ $\mathrm{cm}^{3}$ in $72 \%$ of cases in the study by de la Fuente et al. ${ }^{10}$

Both false-positive cases involved GBMs with large necrotic regions. Although we attempted to avoid necrosis when placing the voxel, precontrast series did not always allow adequate recognition of tumor components. Necrotic tissue contains high concentrations of lipids, some of which resonate between 2.0 and $2.9 \mathrm{ppm}^{21}$ Reevaluation of the voxel positioning revealed that the majority of the

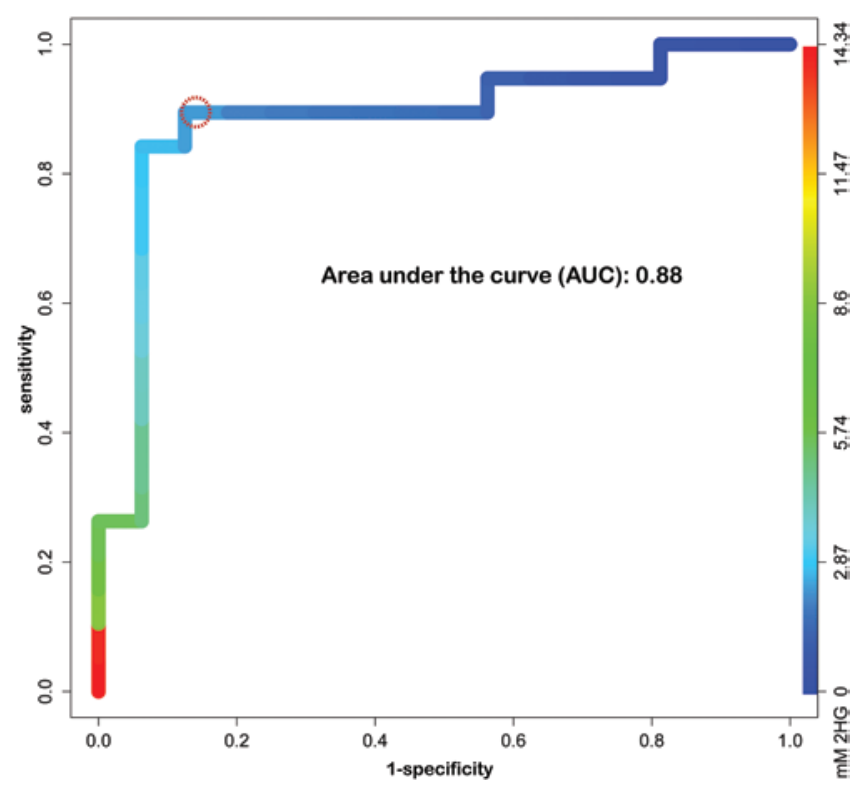

FIG. 4. ROC curve showing the diagnostic performance of 2 HG MRS. The curve's colors illustrate the sensitivity and specificity for different $2 \mathrm{HG}$ values. The red circle indicates the sensitivity and specificity for a cutoff value of $2 \mathrm{mM} 2 \mathrm{HG}$.

voxel was composed of necrotic tissue, and large positive peaks around 1.3 ppm confirmed high lipid concentrations. This was further supported by the reacquisition of a spectrum in one of the cases (GBM with $5.11 \pm 0.31 \mathrm{mM} 2 \mathrm{HG})$, avoiding necrotic tumor tissue guided by previous MRI scans. Wildtype was then, indeed, confirmed by low $2 \mathrm{HG}$ levels $(0.42 \pm 0.05 \mathrm{mM})$. The error of incorrect voxel positioning could have been avoided with smaller voxels and by placement on postcontrast T1-weighted images, where cystic and necrotic tumor components are easy to recognize. This approach has also been recommended by de la Fuente et al. ${ }^{10}$ and could be used in future studies. It is, however, conceivable that different cutoff values for $2 \mathrm{HG}$ concentrations would then have to be chosen. ${ }^{15}$ It is well known that only a minority of GBMs are IDH-mutated (2 of 11 in our study). ${ }^{23}$ Therefore, it is arguable whether the technically more challenging $2 \mathrm{HG}$ MRS in extensively necrotic and enhancing lesions, typical for GBMs, might not be necessary in the first place. Lower patient age as an inclusion criterion would probably have excluded these primary GBMs, but it would also have eliminated important negative control samples from the study. Moreover, other metabolites, such as $\gamma$-aminobutyric acid, glutamate, and glutamine, resonate between 2.2 and $2.4 \mathrm{ppm}$, and their signals may interfere with $2 \mathrm{HG}$ estimation, giving rise to false-positive results. Lastly, to exclude additional mutations, gene sequencing was performed on these 2 cases.

We had 2 false-negative IDH mutations in our series, although the MRS data quality from both patients were acceptable (FWHM: 8.96 and $8.04 \mathrm{~Hz}$, respectively). In 1 case (a Grade III astrocytoma), large parts of the voxel were occupied by a cystic component, whereas the other tumor (a Grade II astrocytoma) was characterized by a very low proliferation rate of $<6 \%$ (detected by Ki 67). 

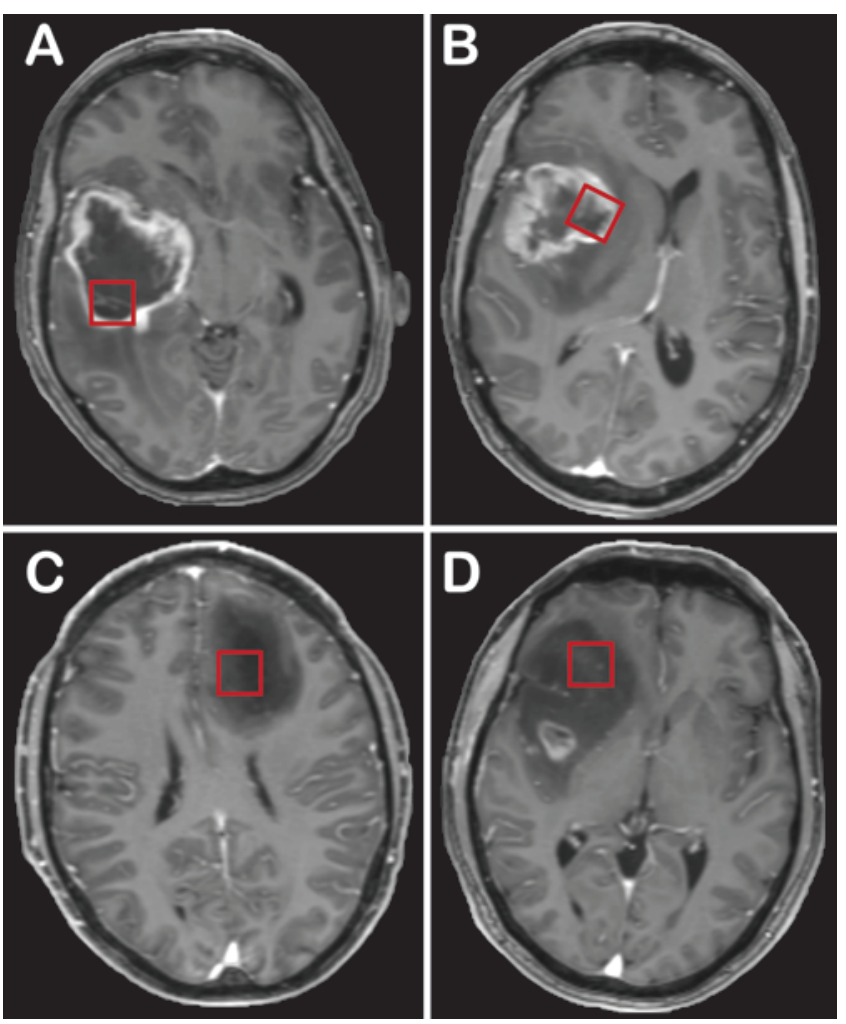

FIG. 5. Axial postcontrast T1-weighted MR images and the corresponding MRS voxels (red squares) of the false-positive ( $\mathrm{A}$ and $\mathrm{B}$ ) and falsenegative ( $C$ and $D$ ) cases. Figure is available in color online only.

In both situations, the tumor cell density and therefore 2HG concentrations might have been under the detectable limit, probably due to sampling from areas with very low tumor cell density. Previous studies have highlighted the relationship between tumor cell density and $2 \mathrm{HG}$ levels, ${ }^{10}$ an important premise for $2 \mathrm{HG}$ MRS being used as a tool for monitoring treatment response and tumor progression. To avoid voxel positioning in regions of low proliferation and consequential false-negative results, $2 \mathrm{HG}$ MRS could be combined with other advanced MRI methods singling out the most aggressive part of the tumor. De la Fuente et al. ${ }^{10}$ suggested using diffusion-weighted MRI and minimum apparent diffusion coefficient (ADC) maps to identify areas with high cellularity. ADC values are, however, known to be influenced by a number of other factors, and the combination of complementary techniques might be meaningful. Voxel positioning can, for example, be improved by using perfusion-weighted MRI, ${ }^{13}$ which is already well established in many imaging departments, or by implementing a fast version of diffusion kurtosis imaging that recently has shown promise in detecting highcellularity tumor components. ${ }^{20}$ Moreover, high-resolution multivoxel MRS techniques may obviate the necessity of precise voxel positioning.

From a clinical point of view, the ability of $2 \mathrm{HG}$ MRS to differentiate glioma from other solitary tumors or nonmalignant brain lesions is of major relevance. Nonspecific findings on conventional MRI sequences or even more advanced imaging methods are a common dilemma. In- cluding $2 \mathrm{HG}$ MRS in the diagnostic process might allow for more rational clinical management of patients with brain lesions. In our small series, $2 \mathrm{HG}$ positivity led to faster decision-making in 2 cases of Grade II astrocytoma, in which conventional MRI demonstrated lesions of unknown significance. In both cases a watch-and-wait approach with regular MRI scans would otherwise have been chosen. $2 \mathrm{HG}$ negativity in the case of autoimmune encephalitis precluded the possibility of a glioma, thereby avoiding unnecessary biopsies.

We evaluated a relatively small number of cases, and this can be regarded as a limitation of our study. Extending the study duration would be likely to improve our results, as we have noticed a considerable learning curve with respect to positioning the MRS voxel and avoiding necrosis, cysts, microhemorrhages, and so on. A further limitation is that we accepted the previously published threshold of $2 \mathrm{mM}$ for IDH mutations, ${ }^{8,9}$ which might not be adequate for our MRI system. In our data set, the sensitivity was $89.5 \%$ and the specificity $81.3 \%$ using this threshold. In fact, a different cutoff was chosen in the study by de la Fuente et al., ${ }^{10}$ and the 2 HG MRS sensitivity is obviously likely to be affected by different thresholds. Moreover, we defined a CRLB of $<20 \%$ and a FWHM of $<10 \mathrm{~Hz}$ as being diagnostic, but clear cutoffs have not been established and affect the sensitivity of this technique. Larger and preferably multicenter studies are necessary to establish more reliable thresholds.

\section{Conclusions}

In conclusion, our results suggest that $2 \mathrm{HG}$ MRS is a useful supplement to clinical tumor MRI protocols. The technique has the potential to narrow differential diagnosis in ambiguous preoperative cases and may be a promising tool for treatment monitoring. If conventional MRI sequences suggest, however, a primary GBM, where IDH mutations are extremely rare, 2HG MRS is not only technically more challenging, but might also not be necessary. Sampling tumor regions with low levels of proliferation can cause false-negative results. In suspected low-grade gliomas it might therefore be reasonable to resample in different regions with an adjusted voxel size and more measurements, possibly at the expense of other MRI sequences, balancing against prolonged imaging duration. The voxel positioning requires some experience and could, at least during the implementation phase, benefit from consensus decisions by the neuroradiologist and neurosurgeon. Although high-resolution multivoxel MRS, optimal protocol design, and the integration of advanced imaging techniques might have the potential to increase the sensitivity and specificity, we propose that this method is of great value for primary diagnosis and treatment assessment, especially in low-grade gliomas.

\section{Acknowledgments}

Funding for this study was provided by the Danish Ministry of Science, Innovation, and Education (Dr. Tietze and Dr. Østergaard); a Central Region Denmark Research Grant (Dr. Tietze); the Cancer Prevention Research Institute of Texas (Grant RP130427, Dr. Choi); Council of Internationalisation and Medical Innovation, Aarhus University Hospital (Dr. von Oettingen). 


\section{References}

1. Ahmadi R, Stockhammer F, Becker N, Hohlen K, Misch M, Christians A, et al: No prognostic value of IDH1 mutations in a series of 100 WHO grade II astrocytomas. J Neurooncol 109:15-22, 2012

2. Andronesi OC, Kim GS, Gerstner E, Batchelor T, Tzika AA, Fantin VR, et al: Detection of 2-hydroxyglutarate in IDHmutated glioma patients by in vivo spectral-editing and 2D correlation magnetic resonance spectroscopy. Sci Transl Med 4:116ra4, 2012

3. Andronesi OC, Loebel F, Bogner W, Marjańska M, Vander Heiden MG, Iafrate AJ, et al: Treatment response assessment in IDH-mutant glioma patients by noninvasive 3D functional spectroscopic mapping of 2-hydroxyglutarate. Clin Cancer Res 22:1632-1641, 2016

4. Beiko J, Suki D, Hess KR, Fox BD, Cheung V, Cabral M, et al: IDH1 mutant malignant astrocytomas are more amenable to surgical resection and have a survival benefit associated with maximal surgical resection. Neuro Oncol 16:81-91, 2014

5. Brat DJ, Verhaak RG, Aldape KD, Yung WK, Salama SR, Cooper LA, et al: Comprehensive, integrative genomic analysis of diffuse lower-grade gliomas. N Engl J Med 372:24812498, 2015

6. Choi C, Ganji S, Hulsey K, Madan A, Kovacs Z, Dimitrov I, et al: A comparative study of short- and long-TE ${ }^{1} \mathrm{H}$ MRS at 3 $\mathrm{T}$ for in vivo detection of 2-hydroxyglutarate in brain tumors. NMR Biomed 26:1242-1250, 2013

7. Choi C, Ganji S, Madan A, DeBerardinis R, Mickey B, Malloy $\mathrm{C}$, et al: Clinical utility of in-vivo MRS measurements of 2-hydroxyglutarate in IDH-mutated gliomas, in 21st Annual Meeting of ISMRM. Concord, CA: ISMRM, 2013, p 509 (Abstract)

8. Choi C, Ganji SK, DeBerardinis RJ, Hatanpaa KJ, Rakheja D, Kovacs Z, et al: 2-hydroxyglutarate detection by magnetic resonance spectroscopy in IDH-mutated patients with gliomas. Nat Med 18:624-629, 2012

9. Choi C, Raisanen JM, Ganji S, Zhang S, McNeil SS, An Z, et al: Prospective longitudinal analysis of 2-hydroxyglutarate magnetic resonance spectroscopy identifies broad clinical utility for the management of patients with IDH-mutant glioma. J Clin Oncol 34:4030-4039, 2016

10. de la Fuente MI, Young RJ, Rubel J, Rosenblum M, Tisnado $\mathrm{J}$, Briggs S, et al: Integration of 2-hydroxyglutarate-proton magnetic resonance spectroscopy into clinical practice for disease monitoring in isocitrate dehydrogenase-mutant glioma. Neuro Oncol 18:283-290, 2016

11. Eckel-Passow JE, Lachance DH, Molinaro AM, Walsh KM, Decker PA, Sicotte H, et al: Glioma groups based on 1p/19q, IDH, and TERT promoter mutations in tumors. N Engl J Med 372:2499-2508, 2015

12. Horbinski C: What do we know about IDH1/2 mutations so far, and how do we use it? Acta Neuropathol 125:621-636, 2013

13. Hu LS, Eschbacher JM, Dueck AC, Heiserman JE, Liu S, Karis JP, et al: Correlations between perfusion MR imaging cerebral blood volume, microvessel quantification, and clinical outcome using stereotactic analysis in recurrent highgrade glioma. AJNR Am J Neuroradiol 33:69-76, 2012

14. Koivunen P, Lee S, Duncan CG, Lopez G, Lu G, Ramkissoon $\mathrm{S}$, et al: Transformation by the (R)-enantiomer of 2-hydroxyglutarate linked to EGLN activation. Nature 483:484-488, 2012

15. Lenkinski RE, Wang X, Elian M, Goldberg SN: Interaction of gadolinium-based MR contrast agents with choline: implications for MR spectroscopy (MRS) of the breast. Magn Reson Med 61:1286-1292, 2009

16. Louis DN, Perry A, Reifenberger G, von Deimling A, Figarella-Branger D, Cavenee WK, et al: The 2016 World Health Organization Classification of Tumors of the Central Nervous System: a summary. Acta Neuropathol 131:803-820, 2016

17. Pope WB, Prins RM, Albert Thomas M, Nagarajan R, Yen $\mathrm{KE}$, Bittinger MA, et al: Non-invasive detection of 2-hydroxyglutarate and other metabolites in IDH1 mutant glioma patients using magnetic resonance spectroscopy. J Neurooncol 107:197-205, 2012

18. Rohle D, Popovici-Muller J, Palaskas N, Turcan S, Grommes C, Campos C, et al: An inhibitor of mutant IDH1 delays growth and promotes differentiation of glioma cells. Science 340:626-630, 2013

19. Schumacher T, Bunse L, Pusch S, Sahm F, Wiestler B, Quandt J, et al: A vaccine targeting mutant IDH1 induces antitumour immunity. Nature 512:324-327, 2014

20. Tietze A, Hansen MB, Østergaard L, Jespersen SN, Sangill $\mathrm{R}$, Lund TE, et al: Mean diffusional kurtosis in patients with glioma: initial results with a fast imaging method in a clinical setting. AJNR Am J Neuroradiol 36:1472-1478, 2015

21. Tugnoli V, Tosi MR, Tinti A, Trinchero A, Bottura G, Fini $\mathrm{G}$ : Characterization of lipids from human brain tissues by multinuclear magnetic resonance spectroscopy. Biopolymers 62:297-306, 2001

22. Verhaak RG, Hoadley KA, Purdom E, Wang V, Qi Y, Wilkerson MD, et al: Integrated genomic analysis identifies clinically relevant subtypes of glioblastoma characterized by abnormalities in PDGFRA, IDH1, EGFR, and NF1. Cancer Cell 17:98-110, 2010

23. Yan H, Parsons DW, Jin G, McLendon R, Rasheed BA, Yuan W, et al: IDH1 and IDH2 mutations in gliomas. N Engl J Med 360:765-773, 2009

24. Zhang N, Zhang L, Qiu B, Meng L, Wang X, Hou BL: Correlation of volume transfer coefficient $\mathrm{K}^{\text {trans }}$ with histopathologic grades of gliomas. J Magn Reson Imaging 36:355-363, 2012

\section{Disclosures}

The authors report no conflict of interest concerning the materials or methods used in this study or the findings specified in this paper.

\section{Author Contributions}

Conception and design: Tietze, von Oettingen. Acquisition of data: Tietze, Parm Ulhøi, Sangill, von Oettingen. Analysis and interpretation of data: Tietze, Choi, Parm Ulhøi, Sangill, LassenRamshad, von Oettingen. Drafting the article: Tietze, Choi, von Oettingen. Critically revising the article: Choi, Mickey, Maher, Parm Ulhøi, Sangill, Lassen-Ramshad, Lukacova, Østergaard. Reviewed submitted version of manuscript: Tietze, Choi, Mickey, von Oettingen. Approved the final version of the manuscript on behalf of all authors: Tietze. Administrative/technical/material support: Tietze, Choi.

\section{Correspondence}

Anna Tietze, Institute of Neuroradiology, Charité-Universitätsmedizin Berlin, Charitéplatz 1, D-10117 Berlin, Germany. email: annatietze@gmail.com. 